\title{
Effect of a restricted geometry on thermal and dielectric properties of $\mathrm{NH}_{4} \mathrm{HSO}_{4}$ ferroelectric
}

\author{
E.A. Mikhaleva $^{1}$, I.N. Flerov ${ }^{1,2}$, A.V. Kartashev ${ }^{1,3}$, M.V. Gorev ${ }^{1,2}$, E.V. Bogdanov ${ }^{1,4}$, \\ V.S. Bondarev ${ }^{1,2}$, L.N. Korotkov ${ }^{5}$, E. Rysiakiewicz-Pasek ${ }^{6}$ \\ ${ }^{1}$ Kirensky Institute of Physics, Siberian Department of RAS, 660036, Krasnoyarsk, Russia \\ ${ }^{2}$ Siberian Federal University, 660074 Krasnoyarsk, Russia \\ ${ }^{3}$ Astafijev Krasnoyarsk State Pedagogical University, 660049 Krasnoyarsk, Russia \\ ${ }^{4}$ Krasnoyarsk State Agrarian University, 660049 Krasnoyarsk, Russia \\ ${ }^{5}$ Voronezh State Technical University, 394026, Voronezh, Russia \\ ${ }^{6}$ Institute of Physics, Wroclaw University of Technology, 50-370 Wroclaw, Poland \\ e-mail: katerina@iph.krasn.ru
}

Heat capacity, thermal dilatation, sensitivity to pressure and permittivity of $\mathrm{NH}_{4} \mathrm{HSO}_{4}$ embedded into glass matrices with a pore size of $320 \mathrm{~nm}$ and $46 \mathrm{~nm}$ were studied. Large difference in the thermal expansion of both glass and ferroelectric leads to a "clamped" state of $\mathrm{NH}_{4} \mathrm{HSO}_{4}$ in nanocomposites and to the phase transition temperatures change. The restricted geometry does not effect on the order of successive transformations in $\mathrm{NH}_{4} \mathrm{HSO}_{4}$ but is accompanied by a significant reduction in entropy of phase transitions. The behavior of DTA-signal and permittivity show the expansion of the temperature range of the ferroelectric phase under hydrostatic pressure.

Keywords Ferroelectric, phase transition, porous glass, nanocomposite PACS: numbers: 62.50.-p; 65.40.-b; 77.80.Bh; 62.23.Pq; 65.80.-g 


\section{Introduction}

Taking into account the intensive development of modern nano- and microelectronic industry, it is not strange that a strong necessity appeared for searching and studying new effective and promising materials [1]. In this way, some nanoscale ferroelectrics, particularly embedded into nanoporous matrices, have been recently widely investigated. The main interest was usually paid to the effect of the type and size of pores on the dielectric properties [2-6]. The phase transition temperature dependence on the pores size of matrices was found increased, decreased or even unchanged in different type of ferroelectrics. Such a phenomenon was explained by different relations between the polarization at the surface and the crystal interior polarization.

In spite of the fact that the study of the thermal properties can provide very important and useful information about entropic parameters as well as interaction between components of the nanoscale composites, heat capacity and thermal dilatation have been studied only sporadically [5, 7-10]. Data regarding the influence of external pressure on the properties of confined ferroelectrics do not exist at all.

In the present paper, we studied heat capacity, thermal dilatation, sensitivity to hydrostatic pressure as well as dielectric properties of $\mathrm{NH}_{4} \mathrm{HSO}_{4}$ embedded into porous boron-silicate glasses with the average pore size of $46 \mathrm{~nm}$ and $320 \mathrm{~nm}$.

Ammonium hydrosulfate is very attractive ferroelectric because, first, it undergoes succession of antiferro- and ferroelectric phase transitions $P-1\left(T_{2}=160 \mathrm{~K}\right) \leftrightarrow P \mathrm{c}\left(T_{1}=271.7 \mathrm{~K}\right)$ $\leftrightarrow P 2_{1} / c$ of the first and second order, respectively $[11,12]$, second, recently the effect of chemical pressure on phase transitions as well as electro- and barocaloric effects in solid solutions $\left(\mathrm{NH}_{4}\right)_{1-\mathrm{x}} \mathrm{Rb}_{\mathrm{x}} \mathrm{HSO}_{4}$ were investigated by us [13]. Moreover, it was possible to suppose large difference in thermal dilatation of solid matrix and embedded ferroelectric, which can lead to the appearance of nonzero intrinsic initial pressure in ferroelectric-glass 
nanocomposite. In order to prepare nanoscale composites, we have used one more important advantage of $\mathrm{NH}_{4} \mathrm{HSO}_{4}$ compared to other water-soluble ferroelectrics associated with a possibility to melt it without decomposition.

\section{Experimental}

In order to be correct analyzing the properties of $\mathrm{NH}_{4} \mathrm{HSO}_{4}$ nano-sized crystals embedded into the glass matrix, it was necessary to study:

1) Thermal properties of glass matrices as well as $\mathrm{NH}_{4} \mathrm{HSO}_{4}$ obtained by melting the compound synthesized from a water solution.

2) Formation of the $\mathrm{NH}_{4} \mathrm{HSO}_{4}$ nanocrystals in the glass matrix.

3) Thermal properties of nanocomposites and $\mathrm{NH}_{4} \mathrm{HSO}_{4}$ in a confined geometry.

The boron-silicate porous glasses were prepared by the same way which was used in [5]. Samples of matrices were cut in a shape of plates with dimensions $10 \times 10 \times 0.5 \mathrm{~mm}^{3}$. The characterization by low-temperature nitrogen sorption and mercury intrusion porosimetry has shown the mean pore diameter 46 and $320 \mathrm{~nm}$ and the porosity about $50 \%$.

The study of $\mathrm{NH}_{4} \mathrm{HSO}_{4}$ obtained from the melt was performed on the "quasiceramic"' samples in the form of pressed tablets $8 \mathrm{~mm}$ in diameter and $1-2 \mathrm{~mm}$ in height, prepared without heat treatment because of the presence of ammonium ion. Nanocomposites containing the ferroelectric component were prepared by immersion of blank porous glasses into the melt of ammonium hydrosulfate for several hours. A mechanical polishing was used to remove micro-crystals from the surfaces of the composite samples. As the result it was found that the mass amount of $\mathrm{NH}_{4} \mathrm{HSO}_{4}$ in composites was about $40 \%$, which corresponds to the filling degree of $70 \%$. The nanocomposites will hereinafter be labeled as Comp 320 and Comp 46 followed by average pore diameter of glass matrices. 
Bulk materials as well as both ferroelectric composites were also characterized using X-ray diffraction technique, which revealed that all samples independent of preparation show the high quality of $\mathrm{NH}_{4} \mathrm{HSO}_{4}$.

Thermal dilatation measurements were performed on the samples above in a helium atmosphere with a heating rate of $3 \mathrm{~K} / \mathrm{min}$ using a NETZSCH model DIL-402C pushrod dilatometer.

The heat capacity studies of the samples examined in dilatometric experiments were carried out by means of a homemade automated adiabatic calorimeter with three screens [14] using discrete as well as continuous heating.

The measurements of dielectric properties were performed in adiabatic calorimeter using an E7-20 immittance metre at different frequencies up to $10^{6} \mathrm{~Hz}$, upon heating/cooling at a rate of about $1.0 \mathrm{~K} / \mathrm{min}$. Silver electrodes were deposited on the samples.

The effect of hydrostatic pressure on the phase transition temperatures in both bulk and nanoconfined $\mathrm{NH}_{4} \mathrm{HSO}_{4}$ was studied by measuring permittivity and/or differential thermal analysis (DTA) signal. Measurements were carried out in a piston-cylinder type vessel associated with a pressure multiplier.

\section{Results and discussion}

Figure 1 shows the temperature dependences of the linear thermal expansion coefficient $\alpha$. One can see that the low temperature anomaly associated with the first order phase transition $P-1 \rightarrow P \mathrm{c}$ was reliably detected in all samples under study. The decrease of the pore size was accompanied by rather strong decrease of the $T_{2}$ value only in Comp 46 $(154.5 \pm 2 \mathrm{~K})$ whereas in Comp $320(165 \pm 2 \mathrm{~K})$ this temperature was changed only a few compared to bulk $\mathrm{NH}_{4} \mathrm{HSO}_{4}(165.5 \pm 1 \mathrm{~K})$. A stronger change was observed in the volumetric strain, $\Delta V / V=3(\underline{l} \underline{l} / l)$, at the $P-1 \rightarrow P \mathrm{c}$ phase transition point: $\mathrm{NH}_{4} \mathrm{HSO}_{4}(-2.4 \%) \rightarrow$ Comp $320(-0.25 \%) \rightarrow$ Comp $46(-0.15 \%)$ 
The anomalous behavior of $\alpha$ associated with the second order transformation $P \mathrm{c} \rightarrow$ $P 2_{1} / \mathrm{c}$ was found strongly smeared in both composites (Inset in Figure 1). The phase transition temperature $T_{1}$ determined as the temperature corresponding to the middle of the stepwise change in expansion coefficient decreases by restricted geometry: $\mathrm{NH}_{4} \mathrm{HSO}_{4}(273 \pm 1 \mathrm{~K}) \rightarrow$ Comp $320(269 \pm 2 \mathrm{~K}) \rightarrow$ Comp $46(258 \pm 3 \mathrm{~K})$.

The results of measurements far from phase transition points show rather large distinction between $\alpha$ values in bulk $\mathrm{NH}_{4} \mathrm{HSO}_{4}\left(\sim 50 \cdot 10^{-6} \mathrm{~K}^{-1}\right)$, nanocomposites $\left(\sim 20 \cdot 10^{-6} \mathrm{~K}^{-}\right.$ ${ }^{1}$ ) and empty glasses $\left(\sim 3 \cdot 10^{-6} \mathrm{~K}^{-1}\right)$ (Figure 1). Such a relation proves that a temperature elevating leads to an appearance of the mechanical stress between the matrix and the ferroelectric component embedded in pores is increased. However, upon heating in a region of $P-1 \leftrightarrow P$ c transformation, strong volume reduction of $\mathrm{NH}_{4} \mathrm{HSO}_{4}$ leads to a decrease in mechanical stress. Such two phenomena can be considered as the pressure effect on ammonium hydrosulfate component from the walls of pores, which is decreased above $T_{2}$. Thus, this is one of the reasons leading to the change of the phase transition temperatures in $\mathrm{NH}_{4} \mathrm{HSO}_{4}$ in restricted geometry.

The experimental results of the heat capacity $C_{\mathrm{P}}(T)$ measurements on bulk $\mathrm{NH}_{4} \mathrm{HSO}_{4}$ and two composites are presented in Figure 2a. All samples demonstrate two anomalies of $C_{\mathrm{P}}(T)$ associated with the successive phase transitions. The $T_{1}$ values observed in calorimetric experiments are close to those found in thermal dilatation measurements. On the other hand, the temperature $T_{2}$ in all samples determined from the $C_{\mathrm{P}}(T)$ dependences is lower on $4-6 \mathrm{~K}$. The reason of such relation between $T_{1}$ as well as $T_{2}$ values determined by different experimental techniques is associated with the second and strong first order phase transitions, respectively. In the latter case large thermal hysteresis in combination with high heating rate (3 $\mathrm{K} / \mathrm{min}$ ) in the measurement of $\alpha(T)$ has led to more higher value of $T_{2}$. 
In contrast to $[5,7]$ we did not observe any peculiarities in the heat capacity behavior proving the change of the $P \mathrm{c} \leftrightarrow P 2_{1} / \mathrm{c}$ phase transition order with the pores size decrease.

The strong size effect on the entropy of phase transitions was detected only in Comp 46 (Figure 2b). The values of the total entropy $\Sigma \Delta S_{\mathrm{i}}=\Delta S_{2}+\Delta S_{1}$ associated with the succession of phase transitions $P-1 \leftrightarrow P \mathrm{c} \leftrightarrow P 2_{1} / c$ are close to each other in both bulk $\mathrm{NH}_{4} \mathrm{HSO}_{4}(7.5 \pm 0.6 \mathrm{~J} / \mathrm{mol} \cdot \mathrm{K})$ and Comp $320(6.8 \pm 1.0 \mathrm{~J} / \mathrm{mol} \cdot \mathrm{K})$. At the same time the decrease of the pores size from $320 \mathrm{~nm}$ down to $46 \mathrm{~nm}$ leads, first, to smearing the entropy jump at $T_{2}$ and, second, to a significant decrease of $\Sigma \Delta S_{\mathrm{i}}=(5.0 \pm 0.5 \mathrm{~J} / \mathrm{mol} \cdot \mathrm{K})$ value. Since according to the thermodynamic theory [15], the entropy of the ferroelectric phase transition, $\Delta S$, is proportional to the square of the polarization, $P^{2}$, strong reduction in its value can be associated with the $P$ decrease in $\mathrm{NH}_{4} \mathrm{HSO}_{4}$ component embedded in the small pores.

Figure 3 shows the temperature dependences of the permittivity measured upon heating. The stepwise dielectric anomaly associated with the low temperature phase transition $P-1 \leftrightarrow P$ c was reliably detected at low $(1 \mathrm{kHz})$ and high $(1 \mathrm{MHz})$ frequencies for bulk $\mathrm{NH}_{4} \mathrm{HSO}_{4}$ as well as both nanocomposites (Insets in Figures $3 \mathrm{a}, \mathrm{b}, \mathrm{c}$ ). The $\varepsilon$ anomaly at high temperature transformation $P \mathrm{c} \leftrightarrow P 2_{1} / \mathrm{c}$ was observed at both frequencies in ammonium hydrosulfate (Figure 3a) and Comp 320 (Figure 3b) whereas Comp 46 showed small peak only at $1 \mathrm{MHz}$ (Figure $3 \mathrm{c}$ ). The change of the $T_{1}$ and $T_{2}$ values in composites determined from $\varepsilon(T)$ dependences are comparable with those observed in calorimetric measurements. In spite of the smearing $\varepsilon$ anomalies in composites, the peak-like and stepwise behavior of permittivity at $T_{1}$ and $T_{2}$, respectively, at different frequencies proves that the order of both transformations is kept in all samples studied.

The surprising results were obtained in the study of the hydrostatic pressure effect on the phase transition temperatures (Figure 4). First of all we performed the DTA(T) and $\varepsilon(T)$ measurements on two pressed tablets of $\mathrm{NH}_{4} \mathrm{HSO}_{4}$ obtained from a water solution as well as 
from the melt which revealed the same values of baric coefficients $\left(\mathrm{d} T_{1} / \mathrm{d} p=86 \mathrm{~K} / \mathrm{GPa}\right.$ and $\mathrm{d} T_{2} / \mathrm{d} p=-120 \mathrm{~K} / \mathrm{GPa}$ ) showing an expansion of the temperature range of the $P \mathrm{c}$ phase under pressure (Figure 4a).

These results are in contradiction with the data by [16, 17], where both $T_{1}$ and $T_{2}$ temperatures in single crystal $\mathrm{NH}_{4} \mathrm{HSO}_{4}$ were found increased with the pressure increase with strong difference in the baric coefficients: $\mathrm{d} T_{1} / \mathrm{d} p=140 \mathrm{~K} / \mathrm{GPa}$ and $\mathrm{d} T_{2} / \mathrm{d} p=765 \mathrm{~K} / \mathrm{GPa}$. It was suggested that intermediate ferroelectric phase disappeared at $p>0.85 \mathrm{GPa}$. However, it is very important to point out that, taking into account the Clausius-Clapeyron equation $\mathrm{d} T / \mathrm{d} p$ $=(\Delta V / V) / \Delta S$, the negative sign of $\mathrm{d} T_{2} / \mathrm{d} p$ obtained by us experimentally in DTA and permittivity studies agrees with the negative sign of the volume strain change $\Delta V / V$ at $T_{2}$ found in measurements of the thermal dilatation (see above). Moreover, the relation between unit cell parameters [11] also shows that upon heating the $P-1 \leftrightarrow P$ c phase transition is accompanied by a large decrease in volume $(\sim 1.5 \%)$.

The results of measurements on nanocomposite samples are shown in Figures $4 \mathrm{~b}$, c. There was not observed any strong systematic change in the value of baric coefficient $\mathrm{d} T_{1} / \mathrm{d} p$ (115 K/GPa for Comp 320; $86 \mathrm{~K} / \mathrm{GPa}$ for Comp 46) compared to bulk $\mathrm{NH}_{4} \mathrm{HSO}_{4}$. The negative sign of the $\mathrm{d} T_{2} / \mathrm{d} p$ value is stored in $\mathrm{NH}_{4} \mathrm{HSO}_{4}$ nanoparticles confined to glass matrices but the rate of the $T_{2}$ temperature lowering under pressure decreases: $-88 \mathrm{~K} / \mathrm{GPa}$ for Comp, $-78 \mathrm{~K} / \mathrm{GPa}$ for Comp 46.

In the DTA measurements under pressure on bulk $\mathrm{NH}_{4} \mathrm{HSO}_{4}$ and Comp 320, a significant reduction in the entropy associated with the phase transition at $T_{2}$ was observed/ Such a phenomenon correlates with the decrease of this entropy at the pore size decrease found in calorimetric experiments on Comp 46.

\section{Conclusions}


The study of heat capacity, thermal dilatation, sensitivity to hydrostatic pressure as well as dielectric properties of bulk ammonium hydrosulfate in comparison with composites with $\mathrm{NH}_{4} \mathrm{HSO}_{4}$ embedded from a melt into nanoporous boron-silicate glasses with the average pore size of $320 \mathrm{~nm}$ and $46 \mathrm{~nm}$ showed following features.

1) Large difference in the thermal expansion coefficients of glass matrix and bulk ammonium hydrosulfate is one of the reasons of the phase transition temperatures change in nanoconfined $\mathrm{NH}_{4} \mathrm{HSO}_{4}$.

2) The decrease of the pore size does not effect on the order of both transformations in $\mathrm{NH}_{4} \mathrm{HSO}_{4}$ embedded into porous matrix but leads to a significant decrease of the total entropy change and smearing both the entropy jump and permittivity at the $P-1 \leftrightarrow P \mathrm{c}$ phase transition.

3) The negative sign of baric coefficient $\mathrm{d} T_{2} / \mathrm{d} p$ found in $\mathrm{NH}_{4} \mathrm{HSO}_{4}$ and both composites correlates with the decrease of the unit cell volume upon heating found in the thermal dilatation measurements and X-ray diffraction study [11].

\section{Acknowledgments}

We are grateful to Dr. Maxim Molokeev for X-ray characterization of the samples.

\section{Funding}

The reported study was partially supported by the Russian Foundation for Basic Research (RFBR), research project No. 16-32-00092 mol_a.

\section{References}

1. Kumzerov Y, Vakhrushev S: Nanostructures within porous materials. In: Nalwa HS, eds. Encyclopedia of Nanoscience and Nanotechnology. New-York: American Scientific Publishers; 2003, V. 10: 1-39,

2. Fokin A, Kumzerov Yu, Koroleva E, Naberezhnov A, Smirnov O, Tovar M, Vakhrushev S, and Glazman M: Ferroelectric phase transitions in sodium nitrite nanocomposites. 
J Electroceram. 2009; 22: 270-275.

3. Rogazinskaya OV, Milovidova SD, Sidorkin AS, Popravko NG, Bosykh MA, and Enshina V S: Dielectric Properties of Ferroelectric Composites with TGS Inclusions. Ferroelectrics. 2010: 397: 191-197.

4. Tarnavich V, Korotkov L, Karaeva O, Naberezhnov A, and Rysiakiewicz-Pasek E: Effect of restricted geometry on structural phase transitions in $\mathrm{KH}_{2} \mathrm{PO}_{4}$ and $\mathrm{NH}_{4} \mathrm{H}_{2} \mathrm{PO}_{4}$ crystals. Optica applicata. 2010; 40: 305-309.

5. Cizman A, Marciniszyn T, Enke D, Barascu A, and Poprawski R: Phase transition in $\mathrm{NH}_{4} \mathrm{HSO}_{4}$-porous glasses nanocomposites. J Nanopart Res. 2013; 15: 1756.

6. Baryshnikov SV, Milinskiy AYu, Charnaya EV, Bugaev AS, and Samoylovich MI:

Dielectric studies of ferroelectric $\mathrm{NH}_{4} \mathrm{HSO}_{4}$ nanoparticles embedded into porous matrices. Ferroelectrics. 2016; 493: 85-92.

7. Cizman A, Marciniszyn T, and Poprawski R: Pressure effect on the ferroelectric phase transition in nanosized $\mathrm{NH}_{4} \mathrm{HSO}_{4}$. J Appl Phys. 2012; 112: 034104.

8. Kutnjak Z, Vodopivec B, Blinc R, Fokin AV, Kumzerov YA, and Vakhrushev SB:

Calorimetric and dielectric studies of ferroelectric sodium nitrite confined in a nanoscale porous glass matrix. J. Chem Phys. 2005;123: 084708.

9. Kumzerov YuA, Kartenko NF, Parfen'eva LS, Smirnov IA, Fokin AV, losewicz D, Misiorek H, and Jezowski A: Capacity and thermal conductivity of a nanocomposite chrysolite asbestos-KDP $\left(\mathrm{KH}_{2} \mathrm{PO}_{4}\right)$. Phys. Solid State. 2011; 53: 1099-1103.

10. Rysiakiewicz-Pasek E, Poprawski R, Polanska J, Urbanowicz A, and Sieradzki A: Properties of porous glasses with embedded ferroelectric materials. J. Non-Crystalline Solids. 2006; 352, 4309-4314.

11. Pepinsky R, Vedam K, Okaya YS, and Hosino S: Ammonium hydrogen sulfate: a new ferroelectric with low coercive field. Phys Rev. 1958; 111: 1508-1510. 
12. Flerov IN, Zinenko VI, Zherebtsova LI, Iskornev IM, and Blat DCh: Study of phase transitions in ammonium hydrosulfate. Izvestiya AN USSR (seriya fizicheskaya) 1975; 39: $752-757$.

13. Mikhaleva EA, Flerov IN, Bondarev VS, Gorev MV, Vasiliev AD, and Davydova TN:

Phase Transitions and caloric effects in ferroelectric solid solutions of ammonium and rubidium hydrosulfates. Phys. Solid State.2011; 53: 510-517

14. Kartashev AV, Flerov IN, Volkov NV, and K.A. Sablina: Adiabatic calorimetric study of the intense magnetocaloric effect and the heat capacity of $\left(\mathrm{La}_{0.4} \mathrm{Eu}_{0.6}\right)_{0.7} \mathrm{~Pb}_{0.3} \mathrm{MnO}_{3}$.

Phys. Solid State. 2008; 50: 2115-2120.

15. Lines ME and Glass AM: Principles and Application of Ferroelectrics and Related Materials. Oxford: Clarendon Press; 1977.

16. Polandov IN, Mylov VP, and Strukov BA: On $\mathrm{p}-\mathrm{T}$ diagram of ferroelectric crystal $\mathrm{NH}_{4} \mathrm{HSO}_{4}$. Fizika Tverdogo Tela. 1968; 10: 2232-2234.

17. Gesi K. and Ozawa K: Pressure-temperature phase diagram of ferroelectric ammonium bisulfate $\mathrm{NH}_{4} \mathrm{HSO}_{4}$. J. Phys. Soc Japan. 1977; 43: 563-569. 


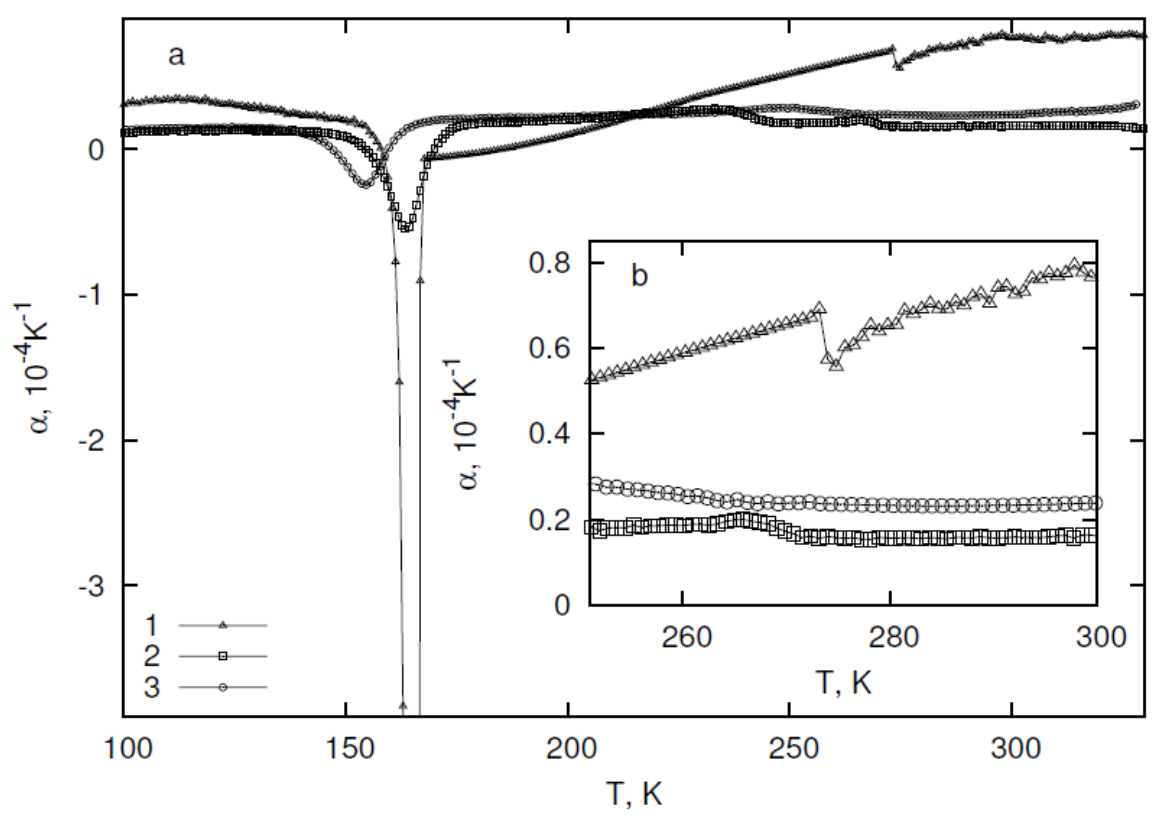




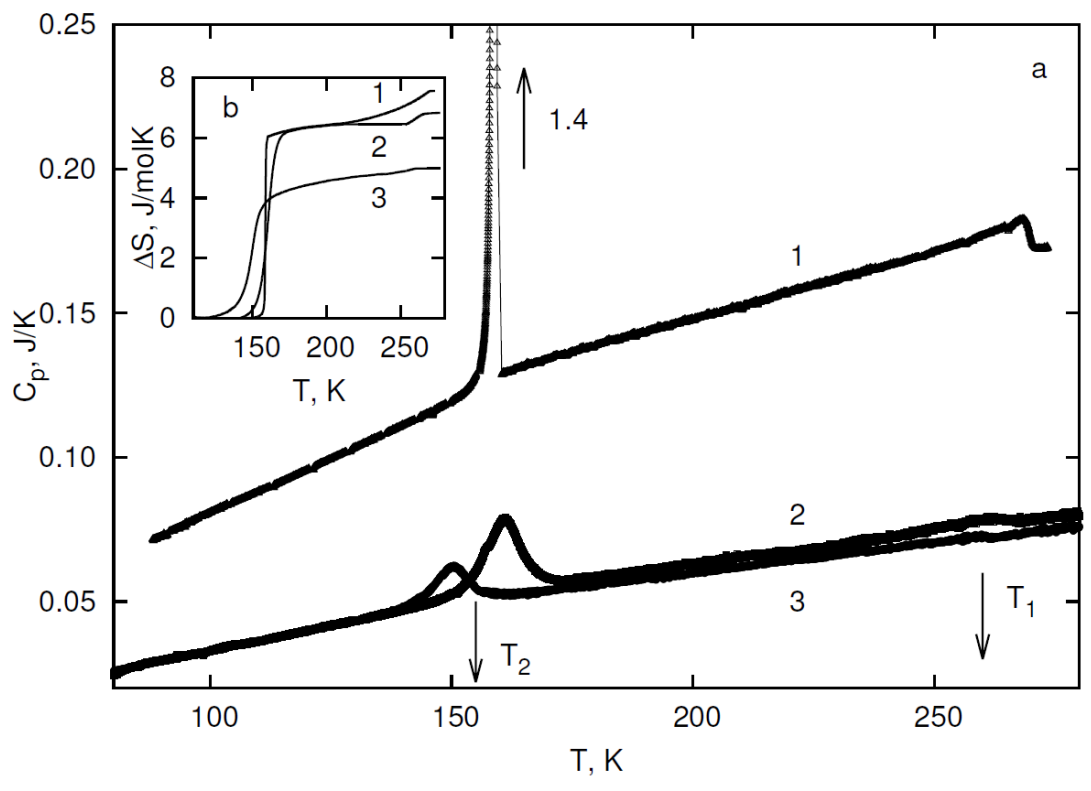



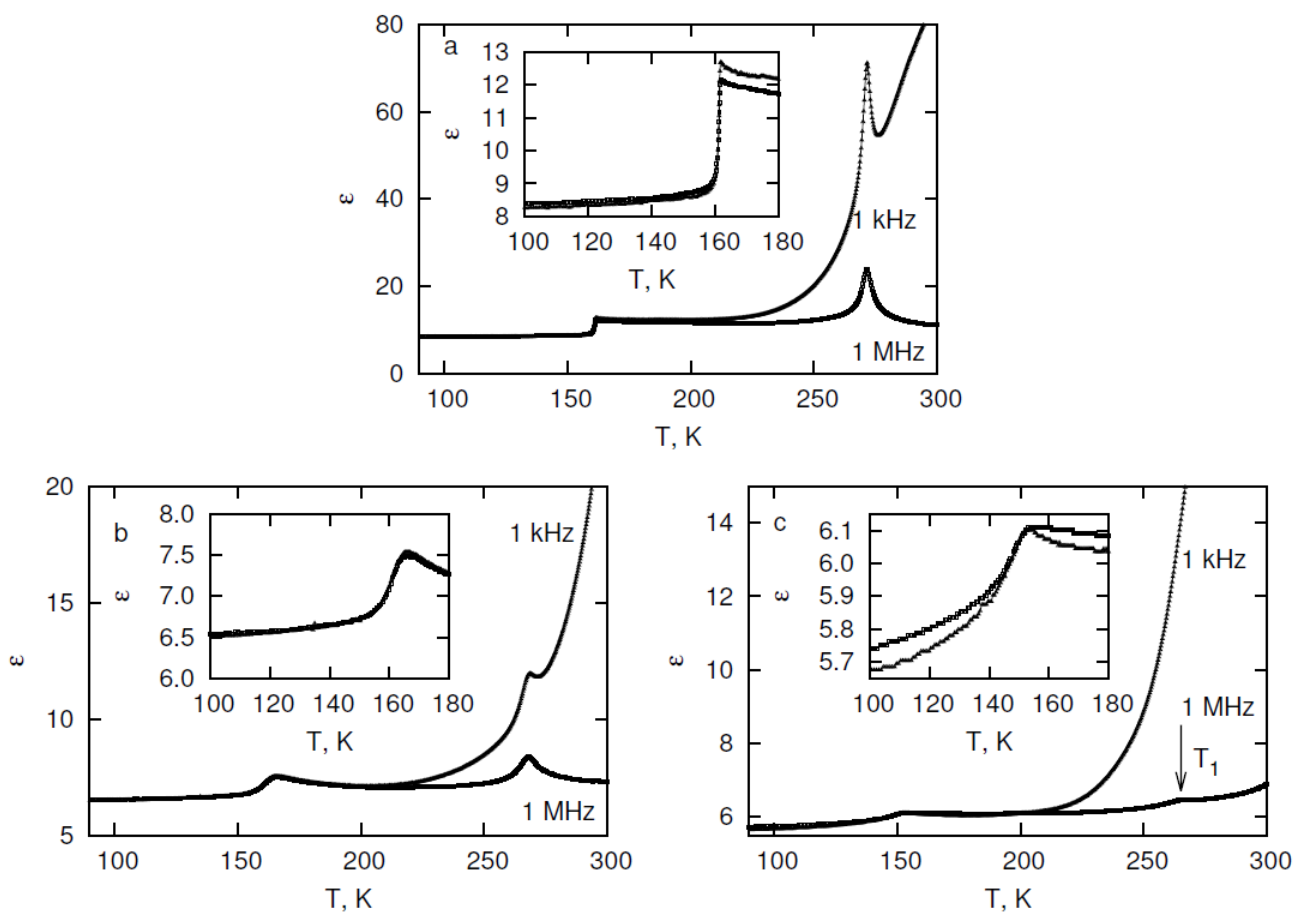

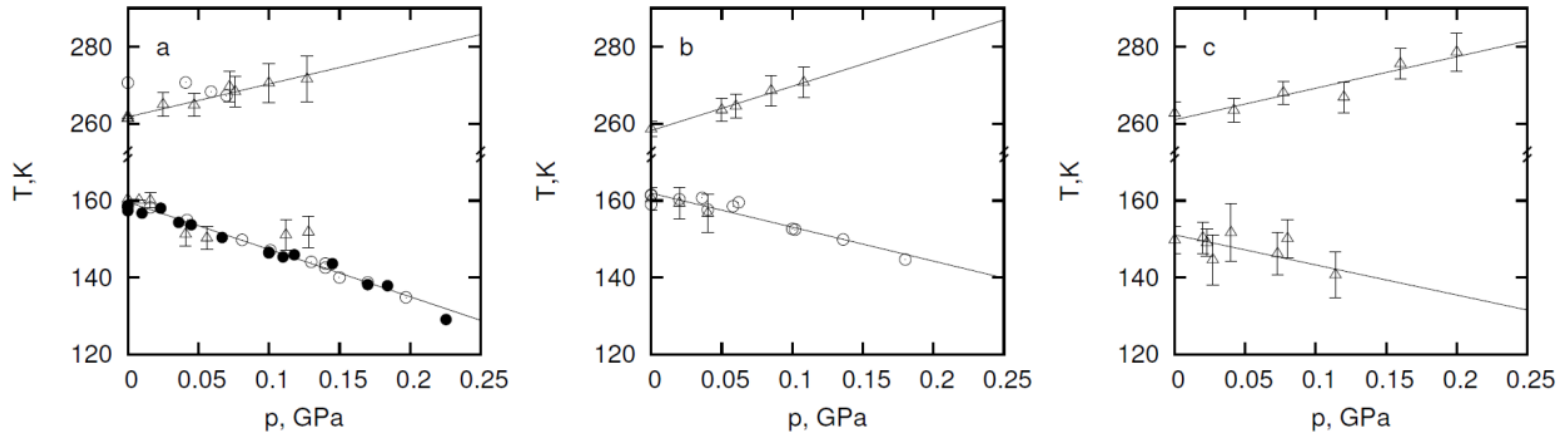


\section{Figure Captions}

Figure 1. Temperature dependences of the linear thermal expansion coefficient $\alpha$ in a wide temperature region (a) and near the $P 2 /{ }_{1} \mathrm{c} \leftrightarrow P \mathrm{c}$ phase transition (b). $1-$ bulk $\mathrm{NH}_{4} \mathrm{HSO}_{4}, 2-$ Comp 320, 3 - Comp 46.

Figure 2. Experimental data on the heat capacity measurements on bulk $\mathrm{NH}_{4} \mathrm{HSO}_{4}$ (1), Comp 320 (2) and Comp 46 (3) (a). Molar entropy change at the successive phase transitions (b).

Figure 3. Temperature dependences of permittivity at $1 \mathrm{kHz}$ and $1 \mathrm{MHz}$ for bulk $\mathrm{NH}_{4} \mathrm{HSO}_{4}$ (a), Comp 320 (b), Comp 46 (c). Insets show the permittivity behavior near $T_{2}$.

Figure 4. T-p phase diagrams of bulk $\mathrm{NH}_{4} \mathrm{HSO}_{4}$ (a), Comp 320 (b) and Comp 46 (c). Open and closed circular points - data obtained by DTA on $\mathrm{NH}_{4} \mathrm{HSO}_{4}$ grown from a water solution and the melt, respectively. Triangular points - data of the permittivity measurements. 
Shortened version of the Title

Effect of restricted geometry on $\mathrm{NH}_{4} \mathrm{HSO}_{4}$ 